way that would be impossible in any alternative that is entirely trust organised. Our scheme should also help psychiatrists to retain an overall identity by reminding us that we all share certain basic clinical skills, whatever our speciality within it. It should also help us to retain a say in what we do and what we stand for at a time when others may be only too willing to make these decisions for us.

H. G. MORGan, Emeritus Professor of Mental Health, University of Bristol, Division of Psychiatry, 41 St Michael's Hill, Bristol BS2 8DZ

\section{Are inappropriate admissions a problem?}

Sir: Elwood's (Psychiatric Bulletin, January $1999,23,37-40)$ study of inappropriate admissions suggests junior doctors can recognise when acute in-patient care is not the most appropriate treatment. Despite this, $24 \%$ of all admissions were deemed inappropriate, with the 'ideal' alternative ranging from social service referral to in-patient rehabilitation.

Occupancy rates are often much higher in England and Wales (Ford et al, 1998) than the recommended $85 \%$ (Royal College of Psychiatrists, 1988), making it desirable for patients who do not require acute in-patient care to be diverted to an appropriate alternative or to be discharged from hospital rapidly. As inappropriate admissions occupied only $3 \%$ of all acute inpatient beds in Elwood's study, diversion to more appropriate care, even if it were possible, might have only modest effects on occupancy rates. A much larger problem contributing to high occupancy rates are patients who, while admitted appropriately, have recovered sufficiently to be discharged. In a recent cross-sectional audit of 89 acute in-patients in Tower Hamlets, $23 \%$ were considered by their keyworker as no longer requiring treatment on an acute ward, and of these $25 \%$ were still in-patients three months later.

Focusing on all patients who do not require inpatient care, rather than only those for whom admission may not have been the ideal intervention is probably a better use of audit resources. In practice, however, inappropriate admissions may contribute to high occupancy levels both by occupying beds and diverting staff time from the review of settled patients who no longer require admission.

FORD, R., DURCAN, G., WARNer, L., et al (1998) One-day survey by the Mental Health Act Commission of acute adult in-patient wards in England and Wales. British Medical Journal, 317. 1279-1283.
Royal College of Psychiatrists (1988) Psychiatric Beds and Resources: Factors Influencing Bed Use and Service Planning. Report of a Working Party of the Section for Social and Community Psychiatry of the Royal College of Psychiatrists. London: Gaskell.

RICHARD DUFFETT, Specialist Registrar in General Psychiatry. St Clement's Hospital, 20 Bow Road. London E3 $4 L L$

\section{Anticholinergic treatment}

Sir: We would like to compliment the excellent report on anticholinergic treatment by Williams (Psychiatric Bulletin, January 1999, 23, 22-24). We would like to add a few points about the use of these medications.

Anticholinergic drugs are often considered as 'side-effect medication' by many patients as well as some clinicians. Hence, they are often prescribed inappropriately, unaware of the fact that they can worsen anticholinergic side-effects including dry mouth, dry eyes, dry flushed skin. dilated pupils and blurring of vision. Anticholinergic drugs help relieve tremor and rigidity, but bradykinesia is less likely to be helped. Extrapyramidal side-effects are not manifested during sleep. Hence, a bedtime dose is unnecessary. Moreover, its stimulant effect can impair sleep. Anticholinergic drugs are ineffective in the prevention and treatment of akathisia and neuroleptic malignant syndrome. Anticholinergic drugs not only increase the risk of developing tardive dyskinesia, but also worsen existing tardive dyskinesia.

Anticholinergic drugs can cause psychological dependence. The street value of procyclidine is about $\& 1$. This may partly explain why some patients feign extrapyramidal symptoms to get a continued and/or increased prescription of these drugs.

K. S. GRacious, Senior House Officer in Psychiatry, Priority Care NHS Trust Hospital, Maidstone ME16 9QQ: S. HOPTON, E. SCHIMPF, A. PRASEEDOM, Senior House Officers in Psychiatry, West Suffolk Hospital, Bury St Edmunds IP33 2QZ

\section{Recent audit of people taking lithium}

Sir: We were interested to read Anderson \& Sowerbutt's paper (Psychiatric Bulletin, December $1998,22,740-743)$. We recently carried out an audit of people on lithium ( $n=27$, age range 66-98 years, mean 79.8) under the care of one old age psychiatrist. Fifty-five per cent had unipolar/psychotic depression, $30 \%$ had a bipolar affective disorder and $15 \%$ had a schizoaffective illness. Eleven had lithium for more than five years. Each person (or carer if the carer was 